\title{
Using Bleichenbacher's Solution to the Hidden Number Problem to Attack Nonce Leaks in 384-Bit ECDSA
}

\author{
Elke De Mulder ${ }^{1}$, Michael Hutter ${ }^{1,2 *}$, Mark E. Marson ${ }^{1}$, and Peter Pearson ${ }^{1}$ \\ 1 Cryptography Research, Inc. \\ 425 Market Street, 11th Floor, San Francisco, CA 94105, USA \\ \{elke,mark\}@cryptography.com ppearson@spamcop.net \\ 2 Institute for Applied Information Processing and Communications (IAIK), \\ Graz University of Technology, Inffeldgasse 16a, 8010 Graz, Austria \\ Michael.Hutter@iaik.tugraz . at
}

\begin{abstract}
In this paper we describe an attack against nonce leaks in 384-bit ECDSA using an FFT-based attack due to Bleichenbacher. The signatures were computed by a modern smart card. We extracted the low-order bits of each nonce using a template-based power analysis attack against the modular inversion of the nonce. We also developed a BKZ-based method for the range reduction phase of the attack, as it was impractical to collect enough signatures for the collision searches originally used by Bleichenbacher. We confirmed our attack by extracting the entire signing key using a 5-bit nonce leak from 4000 signatures.
\end{abstract}

Keywords: Side Channel Analysis, ECDSA, Modular Inversion, Hidden Number Problem, Bleichenbacher, FFT, LLL, BKZ.

\section{Introduction}

In this paper we describe an attack against nonce leaks in 384-bit ECDSA [2] running on a modern smart card. The attack has several interesting and novel features. We first identified a leak during the modular inversion of the nonce, and used differential power analysis (DPA) [17] to identify the likely inversion algorithm. Although the algorithm contains conditional branches, they were not exploitable by simple power analysis (SPA). Instead, we extracted a few loworder bits of each nonce using a template-based power analysis attack [8].

Such nonce leaks are commonly attacked by mapping them to a hidden number problem (HNP), and using lattice methods such as LLL [18], BKZ [26], and Babai's nearest plane algorithm [3] to solve the resulting closest vector problem (CVP) or shortest vector problem (SVP). While it might have been possible to

C)IACR 2013. This article is the final version submitted by the author(s) to the IACR and to Springer-Verlag on 04/06/2013. The version published by Springer-Verlag is available at $\langle\mathrm{DOI}\rangle$.

${ }^{*}$ This work was done while the author was with Cryptography Research, Inc. 
use lattice attacks successfully, our initial template attacks only recovered very few bits reliably. We therefore chose to explore Bleichenbacher's approach [4], which given enough signatures can work with small, even fractional, bit leaks. In contrast, current lattice methods require a minimum number of bits to leak, regardless of the number of signatures used.

Bleichenbacher introduced his FFT-based attack in 2000 during an IEEE P1363 Working Group meeting [1]. He used it to attack the pseudorandom number generator (PRNG) specified by the then-existing DSA standard. While the attack required a prohibitive amount of resources and was not considered practical, there was enough concern about it that the PRNG in the standard was modified [22]. Although this method is known to exist by the cryptanalytic community [25, 28], it remains largely undocumented and has been referred to as an "underground" attack [28]. To remedy this we describe the technique in enough detail so that interested parties can continue studying it.

Bleichenbacher's original analysis required millions of signatures in order to reduce the range of certain values so they could be used in a practical inverse FFT. Since we only had about 4000 signatures available, we looked for a different method of range reduction. We developed a BKZ-based method for this phase of the attack, thereby avoiding an impractical data collection step.

We experimentally confirmed our attack methodology by extracting the entire secret key from a 5-bit nonce leak using about 4000 power traces and corresponding signatures. The attack is an iterative process. Each iteration involves the derivation of about 3000 usable points using BKZ, followed by a pass through an inverse FFT. During each iteration a block of the most significant bits (MSBs) of the unknown part of the secret key is recovered. Finally, our simulations show that a 4-bit leak is also exploitable, with a significant increase in required resources and available signatures. Future research should improve these results.

Related Work Many attacks against nonce leaks in DSA and ECDSA have been published. Boneh and Venkatesan [6] started looking at the HNP in 1996. They mapped the HNP to a CVP and used LLL lattice reduction together with Babai's nearest plane algorithm to study the security of the MSBs of the DiffieHellman key exchange and related schemes.

In 1999 (and officially published in 2001), Howgrave-Graham and Smart [13] applied similar techniques to attack 160-bit DSA given multiple signatures with a fixed signing key and knowledge of some bits from each nonce. Experiments using NTL [27] showed they could recover the secret key given 8 bits of each nonce from 30 signatures, but experiments with 4 bits did not succeed.

In [23] Nguyen and Shparlinski gave a provable polynomial-time attack against DSA in which the nonces are partially known, under some assumptions on the modulus and on the hash function. They were able to recover a 160-bit key with only 3 bits of each nonce from 100 signatures, using the NTL as well. They also showed that given improved lattice reduction techniques it should be possible to recover the key with only 2 nonce bits known. In [24] the same authors extended their result to the ECDSA. 
At PKC 2005, Naccache et al. [21] employed glitch attacks to ensure that the least significant bytes of the nonces were flipped to zero, allowing the authors to apply the same lattice techniques to recover keys from real smart cards. Recently, Liu and Nguyen [19] developed a new algorithm which allowed them to recover 160-bit keys with only 2 leaked nonce bits.

Roadmap The paper is organized as follows. Sect. 2 describes how we used templates to extract the low-order bits of each nonce during the inversion step. In Sect. 3 we describe Bleichenbacher's solution to the HNP, followed by a description of the BKZ-based range reduction technique in Sect. 4. We discuss the parameter values used in the attack and some implementation issues encountered in Sect. 5. Finally, we summarize our results in Sect. 6.

\section{Analysis of the Smart Card}

We analyzed a commercially available smart card that implements ECDSA. The card implements the algorithm for both binary and prime field curves, and we focused on the signature generation process with the 384-bit prime field curve.

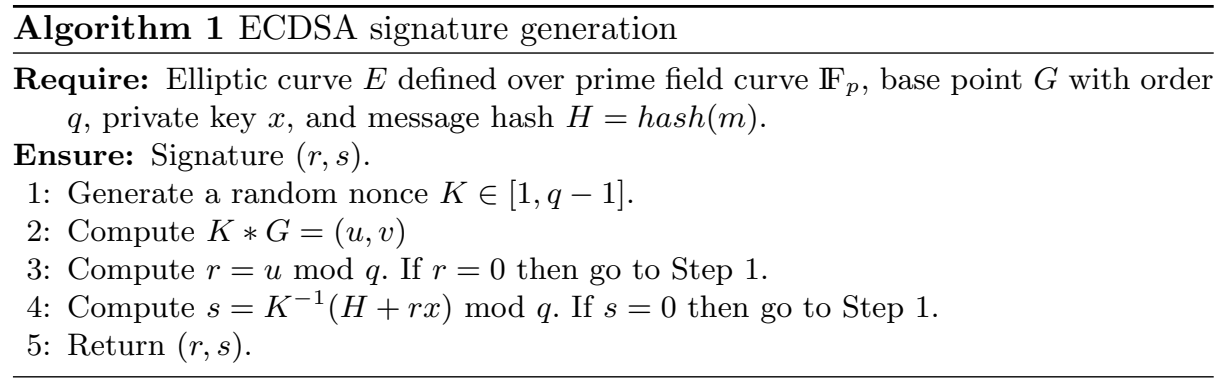

In this section, we describe how the algorithm is implemented on the card. We also describe power analysis results, and identify the several different leakages on the card. Finally, we describe attacks in which we recover either the secret key $x$ or some bits of the nonce $K$. This paper is primarily concerned with the attack in which 7 bits from each nonce are recovered using power analysis against the modular inversion of the nonce in Line 4 of Alg. 1.

\subsection{Description of the Implementation}

Using both reference documentation from the manufacturer and power analysis we determined the card uses the following parameters and techniques.

1. The built-in domain parameters are from ECC-Brainpool [20]. We analyzed the implementation for brainpoolP384r1.

2. Values are represented in Montgomery form for efficient arithmetic. 


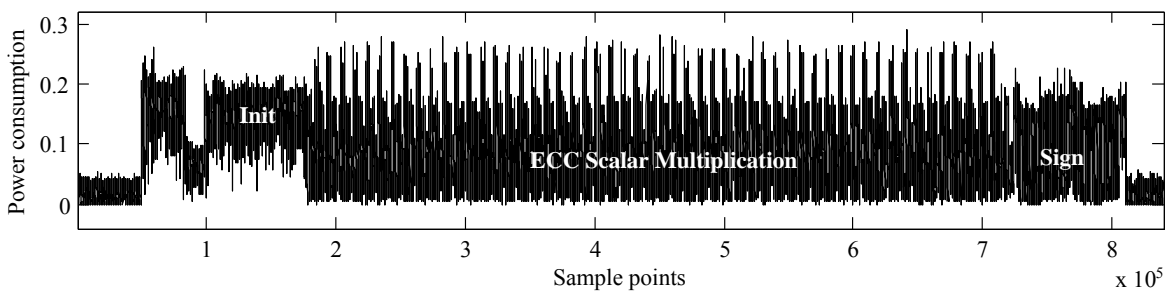

Fig. 1: Power trace of the ECDSA signature generation after post-processing.

3. Curve points are represented in Jacobian projective coordinates.

4. Scalar multiplications take place on the curve twist brainpoolP384t1 and the final result is mapped back to brainpoolP384r1.

5. Scalar multiplications use the signed comb method $[11,12]$ with 7 teeth. The nonces $K$ are represented in a signed basis [15] with 385 bits.

6. The signed basis representation requires that $K$ is odd. If $K$ is even, $q$ is added to it, which does not change the final result of the scalar multiplication.

7. The card stores 64 pre-computed points in memory for point additions, and computes points for subtraction on the fly.

8. $K^{-1} \bmod q$ is computed using a variant of the binary inversion algorithm.

\subsection{Power Measurement Setup}

The power consumption of the smart card was measured using an oscilloscope with a sampling frequency of $250 \mathrm{MS} / \mathrm{s}$. We used two active amplifiers $(+50 \mathrm{~dB})$ and a passive low-pass filter at $96 \mathrm{MHz}$. We also applied several filtering techniques to isolate the data-dependent frequency bands and downconvert them into baseband. These frequencies were identified in a prior device characterization step. Figure 1 shows a single power trace of the entire ECDSA signature generation process after signal processing.

Three main phases can be clearly identified: 1 . The initial phase where the nonce $K$ is generated, 2. the scalar multiplication $K * G$ and 3 . the final phase where the signature $(r, s)$ is calculated.

\subsection{Power Analysis Attacks Against ECDSA}

In this paper we are primarily concerned with attacking the modular inversion of the nonce $K$, in which only a few low-order bits leak. However, we found two other exploitable weaknesses in the card and will discuss them briefly. The first was an SPA leak of the high-order 54 bits of each nonce during scalar multiplication. The second was a DPA attack against the multiplication of the secret by the first half of each signature. The attacks are described in Appendix A.1 and A.2.

Several authors noticed that weak implementations of finite field operations such as modular additions, subtractions or multiplications can lead to successful 


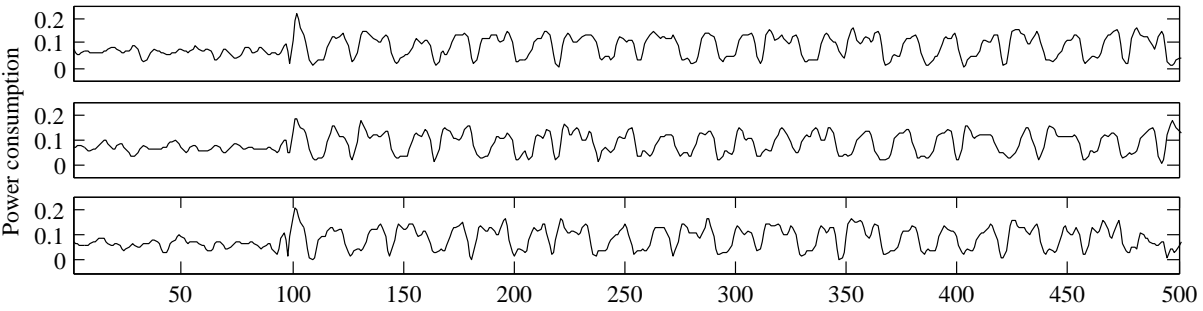

Fig. 2: Power traces during inversion of the first lower-order bits of the nonce.

side channel attacks $[10,16,29,30]$. They proposed eliminating all conditional statements and branches from both software and hardware implementations. This includes final conditional subtractions or reduction steps, which are often found in modular arithmetic operations such as Montgomery multiplication. However, we did not find any publications describing successful template attacks against modular inversions.

The analyzed smart card implements a variant of the binary inversion algorithm. This was identified after a detailed reverse engineering phase in which several intermediate variables of different inversion algorithms were targeted in known-key DPA attacks.

Analysis of the (likely) binary inversion implementation revealed that it does not run in constant time. The execution time depends on the values of both the nonce and the modulus. This is because the algorithm has several conditional branches that depend on the operands. Each branch executes different operations such as modular addition, subtraction, or simple shifts. We were able to construct a set of power consumption templates which represent the power profile for each nonce value. In the next section, we describe the template building and template matching phase in detail and show how we extracted the 7 low-order bits of the nonce with $100 \%$ accuracy.

\subsection{Recovering the Low-Order Bits of the Nonce}

We targeted the low-order bits of the nonce which are processed at the beginning of the modular inversion. To limit the computational complexity we targeted the first 8 bits and generated 256 templates. 1000000 traces were collected: 950000 traces for building templates and 50000 for testing. Some sample traces are shown in Figure 2.

To build the templates we first aligned all the traces at the beginning of the modular inversion. We then sorted the traces by their similarity to the total mean trace using the least mean squares (LMS) algorithm, and excluded all traces which had a low matching factor. A low matching factor occurred in situations when the alignment was not possible and/or high noise was interfering with the measurement. The traces with high matching factor were then partitioned in 256 sets, based on the low-order 8 bits of the corresponding nonces. We then computed the 256 templates by averaging the traces in each partition. 
To increase the success rate during the matching phase, we applied two further enhancements. First, we filtered out all the templates which had a high cross-correlation with other templates. Very similar templates which differed by only a few sample points were not used for the final byte classification. As a result, we only used 102 templates out of 256 . Second, we added a length property for each template. Since the processing time of each nonce byte is not constant but variable during the inversion, the template had to be adapted such that the entire processing time is covered completely. Hence a different number of sample points was used to build the template and to match them with test traces.

\subsection{Analysis Results}

We used 50000 test traces to evaluate the templates. First, we aligned all traces and filtered out 4000 traces due to misalignment and high noise. Second, each trace was matched with all 102 templates using the LMS algorithm. For the classification, we followed a threshold detection approach by rejecting all traces that were below a certain matching threshold. Only those traces with a high quality factor (high correlation with a template) were considered as correctly classified. We obtained 4451 candidates that met all the requirements. For these candidates, all 7 low-order bits were classified correctly, with a success rate of $100 \%$.

Although we were able to extract 7 bits of each nonce in our final template analysis, our earlier attempts recovered only a couple of bits reliably. Hence we decided to implement Bleichenbacher's attack and see if it could succeed with fewer bits. The remainder of this paper describes this attack for a 5-bit leak.

\section{Bleichenbacher's Solution to the Hidden Number Problem}

\subsection{ECDSA Nonce Leaks and the Hidden Number Problem}

We briefly review the basics of exploiting an ECDSA nonce leak by mapping the problem to an HNP. Our notation is mostly consistent with Bleichenbacher's presentation $[4,5]$. Let $q$ be the order of the base point. For $0 \leq j \leq L-1$, where $L$ is the number of signatures, let $H_{j}$ denote the hashes of the messages to be signed, $x$ the private key, $K_{j}$ the ephemeral secret nonces, and $r_{j}$ and $s_{j}$ the two halves of the signatures. Then

$$
\begin{aligned}
s_{j} & =K_{j}^{-1}\left(H_{j}+r_{j} x\right) \bmod q, \\
K_{j} & =s_{j}^{-1}\left(H_{j}+r_{j} x\right) \bmod q .
\end{aligned}
$$

In our case the low-order $b$ bits $(b=5)$ of $K_{j}$, denoted $K_{j, l o}$ were recovered using a template attack. Writing $K_{j}=2^{b} K_{j, h i}+K_{j, l o}$ and rearranging Eq. (1) we get

$$
\begin{aligned}
2^{b} K_{j, h i} & =\left(s_{j}^{-1} H_{j}-K_{j, l o}\right)+s_{j}^{-1} r_{j} x \bmod q, \\
K_{j, h i} & =2^{-b}\left(s_{j}^{-1} H_{j}-K_{j, l o}\right)+2^{-b} s_{j}^{-1} r_{j} x \bmod q .
\end{aligned}
$$


If the original $K_{j}$ are randomly and uniformly generated on $[1, \ldots, q-1]$, then denoting $q_{b}=(q-1) / 2^{b}$, the $K_{j, h i}$ will be randomly and almost uniformly distributed on $\left[0, \ldots,\left\lfloor q_{b}\right\rfloor\right]^{\ddagger}$.

It improves our analysis and attack to center the $K_{j, h i}$ around zero. See Sect. 4 for details. Subtracting $\left\lceil q_{b+1}\right\rfloor$ from both sides of Eq. (2) gives

$$
K_{j, h i}-\left\lceil q_{b+1}\right\rfloor=2^{-b}\left(s_{j}^{-1} H_{j}-K_{j, l o}\right)+2^{-b} s_{j}^{-1} r_{j} x \bmod q-\left\lceil q_{b+1}\right\rfloor .
$$

Denoting $k_{j}=K_{j, h i}-\left\lceil q_{b+1}\right\rfloor, h_{j}=2^{-b}\left(s_{j}^{-1} H_{j}-K_{j, l o}\right)-\left\lceil q_{b+1}\right\rfloor \bmod q$, and $c_{j}=2^{-b} s_{j}^{-1} r_{j} \bmod q$, Eq. (3) becomes

$$
k_{j}=h_{j}+c_{j} x+\alpha_{j} q,
$$

where the $k_{j}$ are almost uniformly distributed on $\left[-\left\lceil q_{b+1}\right\rfloor, \ldots,\left\lfloor q_{b+1}\right\rfloor\right]$ for the appropriate multipliers $\alpha_{j}{ }^{\S}$. We can therefore recover the secret $x$ by solving the following version of the hidden number problem:

Hidden Number Problem: Let $x \in[0, \ldots, q-1]$ be unknown, and suppose we have an oracle which generates random, uniformly distributed $c_{j} \in$ $[1, \ldots, q-1]$ and $k_{j} \in\left[-\left\lceil q_{b+1}\right\rfloor, \ldots,\left\lfloor q_{b+1}\right\rfloor\right]$, computes $h_{j}=\left(k_{j}-c_{j} x\right) \bmod q$, and outputs the pairs $\left(c_{j}, h_{j}\right)$. The goal is to recover $x$.

Lattice-based solutions have been studied extensively and will not be covered here. We only briefly note our own results with these techniques for a 384-bit modulus. Using both the CVP and SVP approaches we were able to attack 6-bit leaks using both LLL and BKZ (fplll v.4.0.1 [7]) for lattice reduction. We could attack 4 and 5-bit leaks with BKZ, but not LLL. The 4-bit attack succeeded twice in 583 trials over a range of $100-200$ points per lattice.

\subsection{Bias Definition and Properties}

Let $X$ be a random variable over $\mathbb{Z} / q \mathbb{Z}$. Bleichenbacher defines the bias of $X$ as

$$
B_{q}(X)=E\left(e^{2 \pi i X / q}\right)=B_{q}(X \bmod q) .
$$

For a set of points $V=\left(v_{0}, v_{1}, \ldots, v_{L-1}\right)$ in $\mathbb{Z} / q \mathbb{Z}$, he defines the sampled bias as

$$
B_{q}(V)=\frac{1}{L} \sum_{j=0}^{L-1} e^{2 \pi i v_{j} / q} .
$$

Some properties of the bias are listed in Lemma 1 below, taken from [5].

Lemma 1. Let $0<T \leq q$ be a bound such that $X$ is uniformly distributed on the interval $[-(T-1) / 2, \ldots,(T-1) / 2]$. Then:

\footnotetext{
${ }^{\ddagger} \operatorname{Pr}\left\{K_{j, h i}==\left\lfloor q_{b}\right\rfloor\right\}$ will be less than for all other values of $K_{j, h i}$ in the interval.

$\S$ We wrote Eq. (4) as an equality because the $k_{j}$ can take on negative values. With this understanding, for the remainder of the paper we will simply write ' $\bmod q$ '.
} 
a. For independent random variables $X$ and $X^{\prime}, B_{q}\left(X+X^{\prime}\right)=B_{q}(X) B_{q}\left(X^{\prime}\right)$.

b. $B_{q}(X)=\frac{1}{T} \frac{\sin (\pi T / q)}{\sin (\pi / q)}$. Hence $B_{q}(X)$ is real-valued with $0 \leq B_{q}(X) \leq 1$.

c. If $X$ is uniformly distributed on the interval $[0 \ldots q-1]$, then $B_{q}(X)=0$.

d. Let $a$ be an integer with $|a| T \leq q$, and $Y=a X$. Then $B_{q}(Y)=\frac{1}{T} \frac{\sin (\pi a T / q)}{\sin (\pi a / q)}$. e. $B_{q}(Y) \leq B_{q}(X)^{|a|}$.

The proofs can be found in Appendix A.3. Some example bias values for $R=$ $T / q=2^{-b}$, for large $q$, are shown in Table 1 below.

Table 1: Example bias values for $R=2^{-b}$

\begin{tabular}{|l|c|c|c|c|c|c|c|c|}
\hline $\mathbf{b}$ & 1 & 2 & 3 & 4 & 5 & 6 & 7 & 8 \\
\hline $\mathbf{B}_{\mathbf{q}}(\mathbf{X})$ & 0.6366198 & 0.9003163 & 0.9744954 & 0.9935869 & 0.9983944 & 0.9995985 & 0.9998996 & 0.9999749 \\
\hline
\end{tabular}

\subsection{Connecting the Hidden Number Problem to the Bias}

In an instance of the HNP, we are given a modulus $q$ and a set of pairs $\left(c_{j}, h_{j}\right), 0 \leq$ $j<L$, and we wish to find the secret and presumably unique $x$ for which the set of values $V_{x}=\left\{h_{j}+c_{j} x \bmod q\right\}_{j=0}^{L-1}$ all fall near 0 or $q$. If they do, then this set of values will show a significantly nonzero sampled bias. Furthermore, for any $w$ different from $x$, we expect that the values $V_{w}=\left\{h_{j}+c_{j} w \bmod q\right\}_{j=0}^{L-1}$ would show a relatively small sampled bias. To see why, for $0 \leq w<q$ let us define $\mathbb{I}$

$$
\begin{aligned}
B_{q}(w) & =\frac{1}{L} \sum_{j=0}^{L-1} e^{2 \pi i\left(h_{j}+c_{j} w\right) / q}=\sum_{t=0}^{q-1}\left(\frac{1}{L} \sum_{\left\{j \mid c_{j}=t\right\}} e^{2 \pi i h_{j} / q}\right) e^{2 \pi i t w / q} \\
& =\sum_{t=0}^{q-1}\left(\frac{1}{L} \sum_{\left\{j \mid c_{j}=t\right\}} e^{2 \pi i\left(h_{j}+c_{j} x\right) / q}\right) e^{2 \pi i t(w-x) / q} \\
& =\sum_{t=0}^{q-1}\left(\frac{1}{L} \sum_{\left\{j \mid c_{j}=t\right\}} e^{2 \pi i k_{j} / q}\right) e^{2 \pi i t(w-x) / q}
\end{aligned}
$$

If $w=x$, then $B_{q}(w)=\frac{1}{L} \sum_{j=0}^{L-1} e^{2 \pi i k_{j} / q}$ is just the sampled bias of the points $k=\left(k_{0}, k_{1}, \ldots, k_{L-1}\right)$. Given a $b$-bit nonce leak $\left(R=2^{-b}\right.$ in Table 1 above $)$ and enough samples, $B_{q}(x)$ will have a value close to 1 , as the points $e^{2 \pi i k_{j} / q}$ are confined to the part of the unit circle with phase $-\pi / 2^{b}<\theta<\pi / 2^{b} . B_{q}(w)$ will be close to zero for any other value of $w$, as the points will be scattered around the unit circle by the $e^{2 \pi i t(w-x) / q}$ terms in Eq. (7).

${ }^{\mathbb{I}}$ We acknowledge the abuse of notation in writing $B_{q}(w)$ instead of $B_{q}\left(V_{w}\right)$, but this is consistent with Bleichenbacher's notes and will simplify the exposition. 
Thus, the bias calculation gives us a way to score putative solutions to the HNP, allowing us to search for the correct value $x$ which maximizes $B_{q}(w)$. Evaluating it for all $w$ in $[0, \ldots, q-1]$ is, of course, impractical for large $q$.

Fortunately, as Bleichenbacher observed [5], it is possible to "broaden the peak" of $B_{q}(w)$ so that values of $w$ near the optimal $x$ will also have large $B_{q}(w)$, allowing us to begin our search by calculating $B_{q}(w)$ for an extremely sparse set of $w$. To see how, note that Eq. (7) shows that $B_{q}(w)$ is a sum of terms $e^{2 \pi i t w / q}$, with frequencies $t / q$. If those frequencies are much smaller than 1 , the peak of $B_{q}(w)$ will be broad, reducing the search work proportionally.

To achieve small $t / q$, we need to work with $\left(c_{j}, h_{j}\right)$ pairs for which the $c_{j}$ are small. Specifically, if we have a bound $C$ such that all the $c_{j}$ satisfy $0 \leq c_{j}<C$, then we can find an approximation to $x$ by searching for the peak value in $B_{q}(w)$ over $n=2 C$ evenly-spaced values of $w$ between 0 and $q$. To evaluate $B_{q}(w)$ over $n$ evenly-spaced values of $w$ between 0 and $q$, set $w_{m}=m q / n, m \in[0, n-1]$, in Eq. (7). Then

$$
\begin{aligned}
B_{q}\left(w_{m}\right) & =\frac{1}{L} \sum_{j=0}^{L-1} e^{2 \pi i\left(h_{j}+\left(c_{j} m q / n\right)\right) / q}=\frac{1}{L} \sum_{j=0}^{L-1} e^{\left(2 \pi i h_{j} / q\right)+\left(2 \pi i c_{j} m / n\right)} \\
& =\sum_{t=0}^{n-1}\left(\frac{1}{L} \sum_{\left\{j \mid c_{j}=t\right\}} e^{2 \pi i h_{j} / q}\right) e^{2 \pi i t m / n}=\sum_{t=0}^{n-1} Z_{t} e^{2 \pi i t m / n}
\end{aligned}
$$

where $Z_{t}=\frac{1}{L} \sum_{\left\{j \mid c_{j}=t\right\}} e^{2 \pi i h_{j} / q}$. The observant reader may recognize the above formula as the inverse FFT of $Z=\left(Z_{0}, Z_{1}, \ldots, Z_{n-1}\right)$. Hence the $B_{q}\left(w_{m}\right)$ can be efficiently computed by first computing the vector $Z$, and then taking the inverse FFT. In practice, we are limited in the number of $B_{q}(w)$ we can evaluate at a given time by the maximum FFT size we can efficiently compute. Hence, we require $\left(c_{j}, h_{j}\right)$ pairs with sufficiently small $c_{j}$. We will discuss range reduction in Sect. 4 . For the next section we will assume the $c_{j}$ are appropriately bounded.

\subsection{Recovering the Secret $\mathrm{x}$ with Bounded $c_{j}$}

Suppose we can compute an $n=2^{N}$-point inverse FFT. Then we can recover the high-order $N$ bits of the $x$ as follows. First zero the vector $Z$. Then:

1. Loop over all $L$ pairs $\left(c_{j}, h_{j}\right)$. For each pair add $e^{2 \pi i h_{j} / q}$ to the appropriate $Z_{t}$, namely $t=c_{j}$.

2. Compute the inverse FFT of $Z$ and find the $m$ for which $B_{q}\left(w_{m}\right)$ is maximal.

3. The most significant $N$ bits of $x$ are $m s b_{N}(x)=m s b_{N}(m q / n)$.

We can repeat the process iteratively to recover the remaining bits of $x$. Let $x=2^{u} x_{h i}+x_{l o}$, where $x_{h i}$ are the known bits previously recovered, and $x_{l o}$ is $u$ bits in length and unknown. We first rewrite Eq. (4) to absorb the known bits $x_{h i}$ into $h_{j}$ :

$$
\begin{aligned}
k_{j} & =\left(h_{j}+c_{j} x\right) \bmod q=\left(\left(h_{j}+2^{u} c_{j} x_{h i}\right)+c_{j} x_{l o}\right) \bmod q \\
& =\left(h_{j}^{\prime}+c_{j} x_{l o}\right) \bmod q .
\end{aligned}
$$


The computation proceeds as before, except we evaluate $B_{q}(w)$ over $n$ evenly spaced values of $w$ between 0 and $2^{u}$, since only $u$ bits remain unknown. Mimicking the previous computation, set $w_{m}=2^{u} m / n, m \in[0, n-1]$ in Eq. (8):

$$
\begin{aligned}
B_{q}\left(w_{m}\right) & =\frac{1}{L} \sum_{j=0}^{L-1} e^{2 \pi i\left(h_{j}^{\prime}+\left(2^{u} c_{j} m / n\right)\right) / q}=\frac{1}{L} \sum_{j=0}^{L-1} e^{\left(2 \pi i h_{j}^{\prime} / q\right)+\left(2 \pi i\left(2^{u} c_{j} m / q n\right)\right)} \\
& =\sum_{t=0}^{n-1}\left(\frac{1}{L} \sum_{\left\{j \mid\left(2^{u} c_{j} / q\right)=t\right\}} e^{2 \pi i h_{j}^{\prime} / q}\right) e^{2 \pi i t m / n}=\sum_{t=0}^{n-1} Z_{t} e^{2 \pi i t m / n}
\end{aligned}
$$

where $Z_{t}=\frac{1}{L} \sum_{\left\{j \mid\left(2^{u} c_{j} / q\right)=t\right\}} e^{2 \pi i h_{j}^{\prime} / q}$. As before, compute the $B_{q}\left(w_{m}\right)$ by taking the inverse FFT of $Z$, and find the $m$ with the maximum value for $B_{q}\left(w_{m}\right)$. The most significant $N$ bits of $x_{l o}$ are $m s b_{N}\left(2^{u} m / n\right)$.

If the $c_{j}$ remain bounded by $C=n / 2$ as the attack proceeds, then $2^{u} c_{j} / q$ would always be zero as soon as $2^{u}<2 q / n$, and the only nonzero coefficient would be $Z_{0}$. Instead, we want $2^{u} c_{j} / q$ to range between 0 and $n / 2$, so we relax the bound on the $c_{j}$ and set $C=n q / 2^{u+1}$ as additional bits of $x$ are recovered.

\section{Range Reduction}

The original $c_{j}$ will be uniformly distributed in $[0, q-1]$ and not nicely constrained as required for the attack described above. The final piece of the puzzle is to find linear combinations of the $c_{j}$ which are in the required ranges, but do not combine too many points. By Lemma 1, combining too many points would attenuate the bias beyond usefulness.

Bleichenbacher's original analysis was for very small, even fractional, bit leaks, and he used millions of signatures, and large FFT sizes. His range reduction strategy was to look for collisions in the high-order bits of the $c_{j}$ and take their differences. For example, if you have $L$ points in $[0, q-1]$, sort them by size, and subtract each point from the next largest one, you get a list of $L-1$ points, which are on average about $\log (L)$ bits smaller. This process can be repeated until the points are in the desired ranges.

In our case we are working with a larger bit leak, but we only have a few thousand signatures, a 384-bit modulus instead of a 160-bit modulus, and a $2^{28}$ point FFT. If we had $2^{30}$ signatures, we could employ the sort-and-difference method to the $c_{j}$ for 12 rounds, taking differences of the corresponding $h_{j}$ as well. This would result in a large number of $c_{j}^{\prime}$ satisfying $0 \leq c_{j}^{\prime}<2^{24}$. The sampled bias for the corresponding points $k_{j}^{\prime}=h_{j}^{\prime}+c_{j}^{\prime} x \bmod q$ would be about $(0.9984)^{2^{12}}=0.0014$, and the attack would succeed.

If we had $2^{20}$ signatures and applied the sort-and-difference method for 18 rounds, the resulting $c_{j}^{\prime}$ would also be in the same range, but the sampled bias

would be about $10^{-182}$, far too small to be useful. With about 4000 signatures available, we looked for another strategy to reduce the range of the $c_{j}$ without reducing the bias too much. 
Given subsets $c_{J}=\left(c_{J, 0}, c_{J, 1}, \ldots, c_{J, d-1}\right)$ of the $c_{j}$, we want to find sets of integer coefficients $A_{J}=\left(a_{J, 0}, a_{J, 1}, \ldots, a_{J, d-1}\right)$, such that $c_{A_{J}}=\left\langle A_{J}, c_{J}\right\rangle \bmod q=$ $\sum_{t=0}^{d-1} a_{J, t} c_{J, t} \bmod q$ satisfies $0 \leq c_{A_{J}}<C$. Applying the $A_{J}$ to Eq. (4) gives $k_{A_{J}}=\left(h_{A_{J}}+c_{A_{J}} x\right) \bmod q$ where the $c_{A_{J}}$ are small enough to be used in the FFT calculation. It is actually a trivial task to find such $c_{J}$ and $A_{J}$. The difficulty lies in finding them subject to the condition that the bias of the resulting $k_{A_{J}}=\left\langle A_{J}, k_{J}\right\rangle \bmod q$ is still large enough to be useful. The most relevant metrics are the $L_{1}$ norm $\left\|A_{J}\right\|_{1}$, and the $L_{\infty}$ norm $\left\|A_{J}\right\|_{\infty}$, which must be sufficiently bounded. Finding bounds $G_{1}$ and $G_{\infty}$ for these norms for which the attack succeeds is discussed in Appendix A.5. Given these bounds, however, we can use BKZ for range reduction and keep only those points which satisfy them.

Consider the lattice spanned by the rows of the following matrix:

$$
\left[\begin{array}{cccccc}
W & 0 & 0 & \cdots & 0 & c_{J, 0} \\
0 & W & 0 & \cdots & 0 & c_{J, 1} \\
\vdots & \vdots & \vdots & \vdots & \ddots & \vdots \\
0 & 0 & 0 & \cdots & W & c_{J, d-1} \\
0 & 0 & 0 & \cdots & 0 & q
\end{array}\right]
$$

The $c_{J, l}$ are randomly chosen from our list of points, and $W$ is a weight factor to balance the reduction of the $c_{J, l}$, and the size of the resulting coefficients. Applying BKZ to the matrix gives

$$
\left[\begin{array}{cccccc}
a_{J, 0,0} W & a_{J, 0,1} W & a_{J, 0,2} W & \cdots & a_{J, 0, d-1} W & c_{A_{J, 0}} \\
a_{J, 1,0} W & a_{J, 1,1} W & a_{J, 1,2} W & \cdots & a_{J, 1, d-1} W & c_{A_{J, 1}} \\
\vdots & \vdots & \vdots & \vdots & \ddots & \vdots \\
a_{J, d, 0} W & a_{J, d, 1} W & a_{J, d, 2} W & \cdots & a_{J, d, d-1} W & c_{A_{J, d}}
\end{array}\right]
$$

where $A_{J, l}=\left(a_{J, l, 0}, a_{J, l, 1}, \ldots, a_{J, l, d-1}\right)$ for $0 \leq l \leq d$ and $c_{A_{J, l}}=\left\langle A_{J, l}, c_{J}\right\rangle \bmod q=$ $\sum_{t=0}^{d-1} a_{J, l, t} c_{J, t} \bmod q$. To simplify notation we will drop the second index $l$ for the rest of the paper.

We want the above lattices to contain points $c_{A_{J}} \in(-C, C)$ for which $\left\|A_{J}\right\|_{1} \leq G_{1}$, and the $\left\|A_{J}\right\|_{\infty} \leq G_{\infty}$. The number of good points per lattice depends not only on those bounds, but also the dimension $d$, the weight $W$, and the BKZ parameters. We determined these experimentally, as described in Appendix A.5. For the first iteration of the attack we used $d=128$, a BKZ blocksize of $20, W=2^{25}, C=2^{28}, G_{1}=325$ and $G_{\infty}=8 . \|$

We can now explain the main reason for centering the $k_{j}$ around zero. It mitigates the reduction of the bias when taking linear combinations. To see why, suppose the range of two independent variables $X$ and $X^{\prime}$ is $[0, \ldots, T-1]$. Then the range of $\pm\left(X \pm X^{\prime}\right)$ is $[-2(T-1), \ldots, 2(T-1)]$. On the other hand, if the range of $X$ and $X^{\prime}$ is $[-(T-1) / 2, \ldots,(T-1) / 2]$, then the range of $\pm\left(X \pm X^{\prime}\right)$ is $[-(T-1), \ldots, T-1]$. Hence if the original $k_{j}$ are centered about zero, then the

\footnotetext{
" Technically, $C$ should be $2^{27}$, but we increased it by a bit in order to find more reduced points, at a cost of decreasing the sampled bias slightly.
} 
$k_{A_{J}}$ in $[0, \ldots, q-1]$ are more densely clustered near 0 and $q$, and therefore have a larger bias. In fact, centering the $k_{j}$ improves the performance of the attack by about a bit in number of leaked nonce bits.

\section{$5 \quad$ Attack Details and Observations}

The attack consists of multiple iterations, in which additional bits of $x$ are recovered in each iteration. Each iteration consists of two phases: range reduction using BKZ, followed by the inverse FFT calculation. The first iteration, with the smallest value for $C$, is the most difficult. As the attack proceeds and $C$ increases, we can find more points $c_{A_{J}} \in(-C, C)$ with smaller coefficient bounds $G_{1}$ and $G_{\infty}$, so fewer points are required for the FFT phase.

We kept a short list of the top 10 scoring candidates from each iteration. We chose to keep 10 candidates based on our experiments with the given bias and the number of points available for the inverse FFT. The correct answer was not always the top candidate, but was always in the top 10, and was the top candidate after the final iteration.

We also used overlapping windows as we successively recovered bits of $x$, keeping the high-order 20 bits out of the 28 recovered. We did this for two reasons. First, the results of the FFT are sometimes off by a few of the low order bits. This is more of an issue when the number of points available is barely sufficient. The second reason is that we used this 8-bit value to round the current approximation of $x$ for the next iteration. We found this rounding essential for getting the correct result in the next iteration. After the next block of bits of $x$ is recovered, the rounding from the previous iteration is undone.

The attack succeeded using 3000 reduced points for each iteration, derived from the original 4000 signatures. However, the work factor and time required was worse than the standard lattice attacks. For the BKZ phase of the first iteration, we used the bounds and lattice parameters discussed above. Each lattice reduction took about 2 minutes, and returned on average 2 usable points. This phase is easy to parallelize, and took about 4 hours to complete on 12 cores. Each $2^{28}$-point FFT took 30 seconds, for a total of 5 minutes. The second iteration was similar, as the increase in $C$ did not improve the BKZ outputs much. The remaining iterations were significantly easier, and the rest of the attack took a few hours to complete.

\section{Conclusions}

In this paper we described an attack against a nonce leak in 384-bit ECDSA running on a smart card. We used a template attack to recover a few low-order bits from each nonce. We then used Bleichenbacher's solution to the HNP, where we had a much larger modulus and far fewer signatures than in his original analysis. Without enough signatures to perform his collision searches, we used $\mathrm{BKZ}$ for range reduction. 
Our attack succeeded against a 5-bit leak with about 4000 signatures, although the time and resources required is worse than what can be done with standard lattice-based attacks. However, our technique will continue to scale with fewer bits. For example, our simulations also show that we could attack a 4-bit leak with 500000 reduced points satisfying $G_{1}=250$ and $G_{\infty}=5$. Finding these points does not appear feasible with the lattice reduction software we used. However, it may be possible to find them using improved implementations such as BKZ 2.0 [9]. There is still a lot of room for improvement in our results, and we hope this paper spurs more research on Bleichenbacher's method.

Acknowledgements. We would like to thank Pankaj Rohatgi and Mike Hamburg for many fruitful discussions and valuable suggestions.

\section{References}

1. Minutes from the IEEE P1363 Working Group for Public-Key Cryptography Standards, November 152000.

2. ANSI X9.62:2005: Public Key Cryptography for the Financial Services Industry, The Elliptic Curve Digital Signature Algorithm (ECDSA), 2005.

3. L. Babai. On Lovász' Lattice Reduction and the Nearest Lattice Point Problem. Combinatorica, 6(1):1-13, 1986.

4. D. Bleichenbacher. On The Generation of One-Time Keys in DL Signature Schemes. Presentation at IEEE P1363 Working Group meeting, November 2000.

5. D. Bleichenbacher. On the Generation of DSA One-Time Keys. Presentation at Cryptography Research, Inc., San Francisco, CA, 2007.

6. D. Boneh and R. Venkatesan. Hardness of Computing the Most Significant Bits of Secret Keys in Diffie-Hellman and Related Schemes. In N. Koblitz, editor, CRYPTO 1996, volume 1109 of LNCS, pages 129-142, 1996.

7. D. Cadé, X. Pujol, and D. Stehlé. fplll-4.0.1 Lattice Reduction Library, 2012.

8. S. Chari, J. R. Rao, and P. Rohatgi. Template Attacks. In B. S. Kaliski Jr., Ç. K. Koç, and C. Paar, editors, CHES 2002, volume 2523 of LNCS, pages 13-28. Springer, 2002.

9. Y. Chen and P. Q. Nguyen. BKZ 2.0: Better Lattice Security Estimates. In ASIACRYPT, volume 7073 of Lecture Notes in Computer Science, pages 1-20. Springer, 2011.

10. G. Hachez and J.-J. Quisquater. Montgomery Exponentiation with no Final Subtractions: Improved Results. In Ç. K. Koç and C. Paar, editors, CHES 2000, volume 1965 of LNCS, pages 91-100. Springer, 2000.

11. M. Hamburg. Fast and Compact Elliptic-Curve Cryptography. IACR Cryptology ePrint Archive 2012: 309.

12. M. Hedabou, P. Pinel, and L. Beneteau. A Comb Method to Render ECC Resistant Against Side Channel Attacks. IACR Cryptology ePrint Archive 2004: 342.

13. N. Howgrave-Graham and N. P. Smart. Lattice Attacks on Digital Signature Schemes. Designs, Codes and Cryptography, 23(3):283-290, August 2001.

14. M. Hutter, M. Medwed, D. Hein, and J. Wolkerstorfer. Attacking ECDSA-Enabled RFID Devices. In M. Abdalla, D. Pointcheval, P.-A. Fouque, and D. Vergnaud, editors, ACNS 2009, volume 5536 of $L N C S$, pages 519-534. Springer, 2009. 
15. M. Joye and M. Tunstall. Exponent Recoding and Regular Exponentiation Algorithms. In B. Preneel, editor, AFRICACRYPT 2009, volume 5580 of $L N C S$, pages 334-349, 2009.

16. P. C. Kocher. Timing Attacks on Implementations of Diffie-Hellman, RSA, DSS, and Other Systems. In N. Koblitz, editor, CRYPTO 1996, volume 1109 of LNCS, pages 104-113. Springer, 1996.

17. P. C. Kocher, J. Jaffe, and B. Jun. Differential Power Analysis. In M. J. Wiener, editor, CRYPTO 1999, volume 1666 of LNCS, pages 388-397, 1999.

18. A. K. Lenstra, H. Lenstra, and L. Lovász. Factoring Polynomials with Rational Coefficients. Mathematische Annalen, 261:515-534, 1982.

19. M. Liu and P. Q. Nguyen. Solving BDD by Enumeration: An Update. In E. Dawson, editor, Topics in Cryptology - CT-RSA 2013, volume 7779 of LNCS, pages 293-309. Springer, 2013.

20. M. Lochter and J. Merkle. Elliptic Curve Cryptography (ECC) Brainpool Standard Curves and Curve Generation. RFC 5639 (Informational), Mar. 2010.

21. D. Naccache, P. Q. Nguyen, M. Tunstall, and C. Whelan. Experimenting with Faults, Lattices and the DSA. In S. Vaudenay, editor, PKC 2005, volume 3386 of $L N C S$, pages 16-28. Springer, 2005.

22. National Institute of Standards and Technology (NIST). FIPS-186-2 (+Change Notice): Digital Signature Standard (DSS), January 2000. Available online at http://www.itl.nist.gov/fipspubs/.

23. P. Q. Nguyen and I. Shparlinski. The Insecurity of the Digital Signature Algorithm with Partially Known Nonces. J. Cryptology, 15(3):151-176, 2002.

24. P. Q. Nguyen and I. Shparlinski. The Insecurity of the Elliptic Curve Digital Signature Algorithm with Partially Known Nonces. Des. Codes Cryptography, 30(2):201-217, 2003.

25. J.-J. Quisquater and F. Koene. DSA Security Evaluation of the Signature Scheme and Primitive. Technical report, Math RiZK, K2Crypt, February 2002.

26. C.-P. Schnorr and M. Euchner. Lattice Basis Reduction: Improved Practical Algorithms and Solving Subset Sum Problems. Mathematical Programming, 66:181-199, 1994.

27. V. Shoup. NTL: A Library for doing Number Theory, 2012.

28. S. Vaudenay. Evaluation Report on DSA. IPA Work Delivery 1002, 2001.

29. C. D. Walter. Montgomery Exponentiation needs no Final Subtractions. Electronics Letters, 35:1831-1832, 1999.

30. C. D. Walter and S. Thompson. Distinguishing Exponent Digits by Observing Modular Subtractions. In D. Naccache, editor, CT-RSA 2001, volume 2020 of LNCS, pages 192-207. Springer, 2001.

\section{A Appendix}

\section{A.1 Targeting the Scalar Multiplication with SPA}

Implemented correctly, the signed comb technique is naturally SPA resistant. If all the required pre-computed points are stored in a lookup table, then the main loop of the scalar multiplication routine is very regular and avoids conditional branches. However, our previous analysis showed that the card only stores the points required for addition. When a point subtraction is needed, the index into the table is computed by complementing the tapped bits of the scalar, and then 
subtracting the accessed point from the current result. The power traces show a prominent spike which is only present when the point subtraction is needed. This SPA leak revealed all 54 higher-order bits of the nonce, after which the lattice-based attack of [13] allowed us to recover the entire secret key $x$ using only 9 power traces.

\section{A.2 Targeting the Private-Key Multiplication with DPA}

When computing the second half of an ECDSA signature, the fixed secret key $x$ is multiplied by the known, varying first half of the signature $r$. This situation is typically vulnerable to standard DPA attacks [14].

We performed the attack by targeting intermediate values during the modular multiplication of $r x \bmod q$. We measured 10000 traces and aligned them using the LMS algorithm. This analysis revealed that the card implements an MSBfirst digit-serial modular multiplication method with a full multiplication of $r x$ followed by modular reduction by division. The high-order 384 bits of the 768-bit intermediate result leak at different positions during the reduction step. Hence our attack proceeded as follows.

First, we defined a search range of 12 bits and targeted the 8th bit. After calculating all possible 4096 hypotheses, we performed a difference of means test that showed peaks for the correct hypotheses. Because multiplication is a linear operation, all bit-shifted variants of the correct hypothesis (multiples and fractions of the correct guess) also showed peaks. This was addressed by assuming the MSB is a one and determining the length of the private key during the DPA attack. The remaining bits of the secret $x$ can be recovered iteratively. In total, we recovered the entire key $x$ in a few hours, where most of the time was spent generating all possible intermediate values.

\section{A.3 Proof of Lemma 1}

a. This formula follows from the fact that the probability distribution of the sum of independent variables is the convolution of the variables' distributions, together with the relationship between Fourier transforms and convolutions.

b. This formula can be computed directly using the standard formula for geometric sequences. The value is real because we centered the distribution of points about zero, and the resulting values on the unit circle are symmetric about the x-axis. Without centering the bias would be complex, with the same absolute value. Also, if $T$ is even, then the formulas still hold, with the shifted points taking on half-integer values.

c. Follows immediately from setting $T=q$ in part b.

d. Same as part b.

e. Write

$$
B_{q}(X)=\frac{1}{T} \frac{\sin (\pi T / q)}{\sin (\pi / q)}=\frac{\frac{\sin (\pi T / q)}{\pi T / q}}{\frac{\sin (\pi / q)}{\pi / q}}
$$


Setting $y=\pi / q$ and $F(y)=\log (\sin (y) / y)$ we want to show that

$$
F(a T y)-F(a y) \leq a(F(T y)-F(y)) .
$$

This will be true if $F$ is concave down. Taking the second derivative gives $F^{\prime \prime}(z)=1 / z^{2}-1 / \sin ^{2}(z)$, which is negative for $z \in(0, \pi)$. Hence Eq. (14) holds and the result is proved.

\section{A.4 Bias Approximation Formulas}

We can find convenient approximations to the formulas in Lemma 1 by taking limits as $q \rightarrow \infty$.

Lemma 2. Suppose $R=T / q$ remains fixed as $q$ varies, with random variables $X_{q}$ uniformly distributed on $[-(T-1) / 2, \ldots,(T-1) / 2]$ for each $q$. Let $Y_{q}=$ $a X_{q}$. Finally define $B_{\infty}(X)=\lim _{q \rightarrow \infty} B_{q}\left(X_{q}\right)$ and $B_{\infty}(Y)=\lim _{q \rightarrow \infty} B_{q}\left(Y_{q}\right)$. Then:

a. $B_{\infty}(X)=\sin (\pi R) / \pi R$.

b. $B_{\infty}(Y)=\sin (a \pi R) / a \pi R$.

Proof. L'Hôpital's rule.

\section{A.5 Finding BKZ Parameters for Range Reduction}

The weights $W$ are required to balance the reduction of the $c_{j}$ with the size of the coefficients. If $W$ is too small, say $W=1$, then the lattice would contain many $c_{A_{J}} \in(-C, C)$ but the coefficients would be too large. On the other hand, if $W$ is too large, say $C \leq W$, the coefficient norms would be smaller, but there would be very few if any $c_{A_{J}} \in(-C, C)$. The values for $W$ which appear to work best are those which are just a few bits smaller than $C$. In our lattices we set $W=2^{\lfloor\log (C)\rfloor-3}$.

We now turn to the question of the coefficient bounds $G_{1}$ and $G_{\infty}$. We need to know for which bounds the attack will work, and how many $\left(c_{A_{J}}, h_{A_{J}}\right)$ pairs are required. Ideally we would run experiments using reduced points output by BKZ. Unfortunately, the lattices which worked best were large, containing 128 or more points. Each lattice reduction took 1-2 minutes, and yielded only a few, if any useful points. This made it impractical to use BKZ outputs of our real data to analyze coefficient bounds.

We therefore analyzed the distribution of coefficients output by BKZ in order to simulate them. For our analysis and attack, we used BKZ with $d=128$, and a blocksize of 20. We randomly populated and reduced lattices, and sorted the $c_{A_{J}}$ based on the bounds $C, G_{1}$ and $G_{\infty}$ they satisfied. Once we had enough $c_{A_{J}}$ for each set of bounds, we examined the distribution of nonzero coefficients. An example is shown in Fig. 3, for $C=2^{28}, G_{1}=325$ and $G_{\infty}=8$. The distribution strongly resembles a normal distribution, and a normal fit also appears to match 
the data.** We therefore modeled the output of BKZ using normal distributions, after getting estimates of the coefficient standard deviations for different sets of bounds.

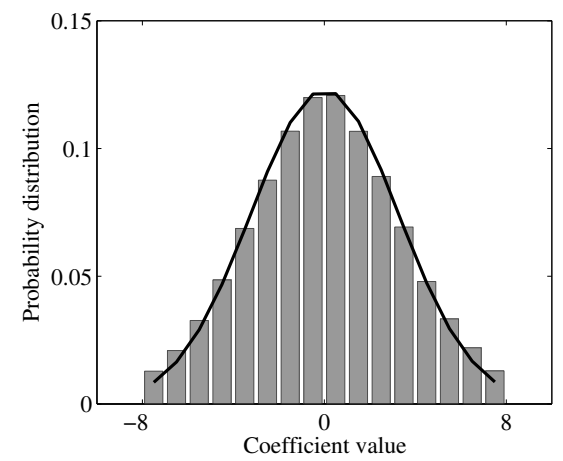

Fig. 3: Distribution and normal fit of the nonzero coefficients output by BKZ.

Note that this was not intended as a rigorous mathematical analysis. We only needed a reasonable model of the coefficient distribution for our simulations. Once we had that, we generated simulated data points $\left(c_{j}, h_{j}\right)$ and coefficients $A_{J}$ with $\left\|A_{J}\right\|_{1} \leq G_{1}$ and $\left\|A_{J}\right\|_{\infty} \leq G_{\infty}$ such that the $c_{A_{J}} \in(-C, C)$. We then performed the FFT phase of the attack in order to determine the number of $\left(c_{A_{J}}, h_{A_{J}}\right)$ pairs required for success. The simulations were accurate, and successfully predicted the number of points required by the attack on actual data. For example, our simulations predicted that for a 5 -bit leak with bounds $G_{1}=325$ and $G_{\infty}=8$, the first phase of the attack would succeed with about 3000 reduced pairs $\left(c_{A_{J}}, h_{A_{J}}\right)$, matching what occurred in the real attack.

\section{A.6 Most Significant vs. Least Significant Bit Leaks}

In [23] the authors noted that depending on the modulus, the most significant bit can carry less information than lower-order bits. This difference can be quantified in terms of the bias. We illustrate this by comparing 5-bit leaks for NIST P-384 and brainpoolP384r1.

The base point for the NIST curve has order $q=0 x F F F F F F F F . .$. , and for the Brainpool curve $q=0 \times 8 \mathrm{CB} 91 \mathrm{E} 82 \ldots$ If the low-order 5 bits leak, then for either prime we get $T=\left\lfloor q / 2^{5}\right\rfloor$ and $R=T / q \approx 2^{-5}$, for a bias of 0.9984 .

If the high-order 5 bits leak, then $T=2^{379}$. For the NIST prime, we still have $R=T / q \approx 2^{379} / 2^{384}=2^{-5}$. Hence the work to attack a 5 -bit leak is the same whether the MSBs or LSBs are recovered. On the other hand, for the Brainpool

\footnotetext{
${ }^{* *}$ Curiously, the coefficient distributions output by LLL were better modeled by geometric distributions.
} 
prime we have $R=T / q \approx 0 \times 8 / 0 \times 8 \mathrm{C}=1 / 17.5$ and a resulting bias of 0.9946 . This is much closer to the value for a 4-bit LSB leak.

Our experiments confirm these calculations. For the NIST prime the work factor for the attack does not depend on whether the MSBs or LSBs are leaked. On the other hand, for the Brainpool prime the work required to attack a 5-bit leak of the MSBs is on par with the work to attack a 4-bit leak of the LSBs. Given the form of the Brainpool prime, about 8/9 of the time the high-order bit of a randomly generated nonce is zero. Hence when the MSBs are leaked, we gain on average very little additional information about the high-order bit. 